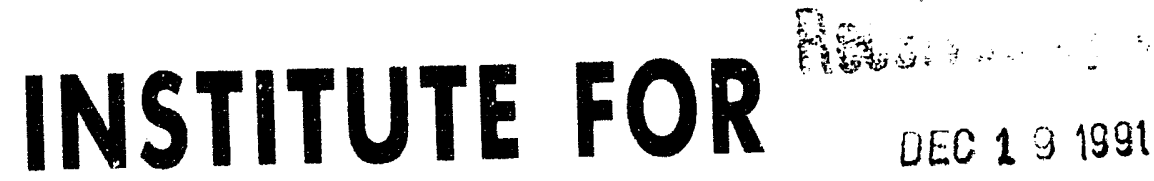 FUSION STUDIES
}

DOE/ET-53088-524

IFSR \#524

Neoclassical Diffusion in a Turbulent Plasma

P. Yushmanov

Kurchatov Institute of Atomic Energy

123182 Moscow, U.S.S.R.

and

Institute for Fusion Studies

The University of Texas at Austin

Austin, Texas 78712

November 1991

THE UNIVERSITY OF TEXAS

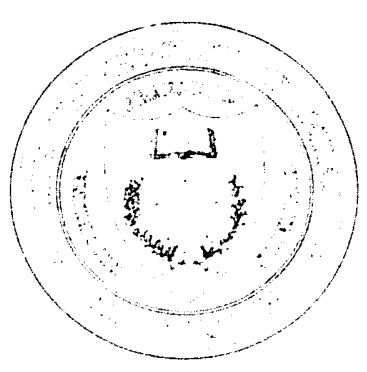

AUSTIN 


\title{
Neoclassical Diffusion in a Turbulent Plasma
}

\author{
P.N. Yushmanov \\ Kurchatov Institute of Atomic Energy \\ 123182 Moscow, U.S.S.R. \\ and \\ Institute for Fusion Studies \\ The University of Texas at Austin \\ Austin, Texas 78712
}

\begin{abstract}
This work describes a new approach to plasma transport where the toroidal drift motion is considered as a perturbation to the fluctuating velocity. Percolation theory is used to determine the scaling of the diffusion coefficient. Several neoclassicai phenomena should persist even when diffusion is enhanced from neoclassical predictions. Numerical simulation results support the theoretical scaling arguments.
\end{abstract}

\section{DISCLAIMER}

This report was prepared as an account of work sponscred by an agency of the United States This report was prepared as an ancer theref, nor any Government. Neither the United States Government nor ans agsumes any legal liability or responsiemployees, makes any warranty, express or implied, or assu information, apparatus, product, or bility for the accuracy, completeness, or usefulness of any information, apparatus, prodect, process disclosed, or represents that its use would not infringe privately owned service by trade name, rademark, ence herein to any specific commercial product, process, or servitu its endorseme it, recommanufacturer, or otherwise does not necessarily constite or any agency thereof. The views

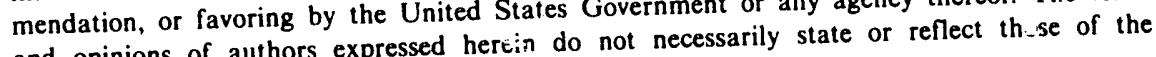
and opinions of authors expressed herein do not
United States Government or any agency thereof. 


\section{Introduction}

Experimentally observed particle and heat fluxes in toroidal devices exceed their neoclassical values about 10-100 times for electrons and 1-10 times for ions. ${ }^{1,2}$ It is commonly agreed that a reason for these anomalous transport processes is turbulent electromagnetic fields that are always present in tokamak discharges. For typical levels of fluctuation the oscillatory velocity of particles due to field perturbations, $\tilde{v}$, exceeds the drift velocity in a toroidal magnetic field, $\tilde{v}$. Therefore deviation of particles from the unperturbed magnetic surfaces is ccntrolled by $\tilde{v}$, not $u_{d}$ and orbits looks quite different from usual neoclassical banana or passing trajectories. This modification of orbit size and form can explain the discrepancy between experimental particle and heat fluxes and neoclassical ones and influence all other neoclassical effects such as boot-strap current or the correction to lon ${ }_{e}$ itudinal conductivity which are known to be close to their neoclassical values. ${ }^{3,4}$ It is found that not all neoclassical effects are destroyed by plasma turbulence, and even if the toroidal drift $u_{d}$ is smaller than $\tilde{v}$, it remains an important characteristic of particle behavior in toroidal systems. The analysis of the mutual influence of turbulent and neoclassical effects is of great interest especially for the ion plasma component for which the experimental discrepancy from neoclassical theory is not too great.

In this paper the following two questions are discussed. How do neoclassical effects (at least some of them) persist under the condition $u_{d}<\tilde{v}$ and how is neoclassical diffusion modified in the presence of high amplitude oscillations. For the purpose of analysis a nonself-consistent approach is used where the electromagnetic field characteristics, including fluctuation amplitudes and spectra are prescribëd and are not influenced by the particles'

motion. For the tokamak plasma turbulence we generally have in mind low frequency drift oscillations with $\omega \sim \omega_{*}=k_{\perp} c T / e B_{0} L_{n},\left(L_{n}=n / \nabla n\right)$ strongly elongated along magnetic 
field lines: $k_{\|}^{-1} \sim q R$ and $k_{\perp}^{-1} \sim \rho_{i}$ ( $\rho_{i}$ being ion Larmor radius).

Particle trajectories with account of motion due to fluctuations and toroidal drifts are considered first. From this analysis it is shown that a small group of particles that move in the direction of toroidal drift, even if $u_{d}<\tilde{v}$, will still contribute to neoclassical effects. This small group of particles also plays a major role in anomalous transport processes and can cause diffusion with coefficients higher than neoclassical ones.

\section{Drift Motion Equations in the Presence of Oscillations}

For the low frequency oscillations guiding center equations describe particle motion. In the toroidal magnetic field of the simplest form

$$
\mathbf{B}=\left(B_{\phi} \hat{\boldsymbol{\phi}}+B_{\theta}(r) \hat{\boldsymbol{\theta}}\right)(1-\varepsilon \cos \theta)
$$

where $\phi$ and $\theta$ are poloidal and toroidal angles, $\varepsilon=r / R$ is the ratio of minor and major radii of the torus, the guiding center drift equations can be written as

$$
\begin{aligned}
& \frac{d z}{d t}=v_{\|}, \\
& \frac{d v_{\|}}{d t}=\frac{e}{m} E_{\|}-\frac{\mu B_{0} \varepsilon}{q R} \sin \theta, \\
& \frac{d \mathbf{r}_{\perp}}{d t}=\frac{c}{B_{0}} \mathbf{E} \times \mathbf{b}+v_{\|} \mathbf{b}+u_{d}(\hat{\boldsymbol{\theta}} \cos \theta+\hat{\mathbf{r}} \sin \theta) .
\end{aligned}
$$

where $\mu=v_{\perp}^{2} / 2 B$ is magnetic moment, $\mathbf{b}=\left(\mathbf{B}_{0}+\tilde{\mathbf{B}}\right) / B_{0}$ is a unit vector along magnetic field, $\mathbf{E}$ and $\mathbf{B}$ are electric and magnetic fields of oscillations, $u_{d}=\left(v_{\perp}^{2}+2 v_{\|}^{2}\right) / 2 \omega_{B i} R$ is the drift velocity in the unperturbed toroidal magnetic field.

Let us estimate the role of each term in equations of motion $(2)-(t)$. To do this we substitute amplitudes of $\mathbf{E}$ and $\mathbf{B}$ that can be obtained from the standard mixing-length 
rule $e^{5}$

$$
\frac{e \tilde{\phi}}{T} \sim \frac{1}{k_{\perp} L_{n}} .
$$

First of all, taking account of typical fluctuation spatial scales $k_{\|}^{-1} \sim q R$ and $k_{\perp}^{-1} \sim \rho_{i}$, it can be easily seen that fluctuations in the longitudinal acceleration is much weaker than $\mu \nabla_{\|} B$ term

$$
\left(\frac{e}{m_{i}} \tilde{E}_{\|}\right)\left(\frac{\mu B_{0} \varepsilon}{q R}\right)^{-1} \sim \frac{\rho_{i}}{\varepsilon L_{n}} \ll 1 .
$$

Therefore the longitudinal particle motion is nearly unaffected by the fluctuating fields and can be integrated separately without taking the perpendicular drift into account. This is why the fraction of the banana trapped particles, that are important for the experimental manifestation of some neoclassical effects (for example, neoclassical correction to longitudinal conductivity), remain the same in the presence of fluctuations.

As the longitudinal equations (2)-(3) can be integrated separately, there is the possibility of representing equation (4) in a form where all terms on the right-hand side are functions of $\mathbf{r}_{\perp}$ and time only. This equation describes the deviation of particle from the unperturbed magnetic field line and can be written in a Hamiltonian form

$$
H\left(\mathbf{r}_{\perp}, t\right)=\widetilde{H}\left(\mathbf{r}_{\perp}, t\right)+u_{d}(x \cos \theta(t)-y \sin \theta(t))
$$

where $\widetilde{H}\left(\mathbf{r}_{\perp}, t\right)$ is the effective fluctuation potential,

$$
\widetilde{H}\left(\mathbf{r}_{\perp}, t\right)=\left(c \tilde{\phi}\left(\mathbf{r}_{\perp}, t\right)+v_{\|}(t) \tilde{A}\left(\mathbf{r}_{\perp}, t\right)\right) / B
$$

$\tilde{\phi}$ and $\tilde{A}$ is the electrostatic and longitudinal magnetic potentials of oscillations, respectively, and $y=\left(\theta \phi / q_{m}\right) r_{m}$ and $x=r-r_{m}$ the perpendicular coordinates and $r_{m}$ the reference magnetic surface.

The fluctuational part of Hamiltonian $\widetilde{H}\left(\mathbf{r}_{\perp}, t\right)$ is a spatially random function with zero average value $\langle\widetilde{H}\rangle=0$, with a characteristic amplitude $H_{0} \equiv\left(\left\langle\widetilde{H}^{2}\right\rangle\right)^{1 / 2} \sim \widetilde{v} / k_{\perp}$ and characteristic time scale $\tau \sim \Delta \omega^{-1} \geq \omega_{*}$. In addition the total Hamiltonian $H\left(\mathbf{r}_{\perp}, t\right)$, given by (7), 
has a time dependence with the frequency $\omega_{t}=v_{\|} / q R$ due to the motion of particles along magnetic field lines. For tokamak drift fluctuation spectra typically $\Delta \omega \leq \omega_{t}$. Therefore the time dependence of the Hamiltonian (7) is generally controlled by the longitudinal motion of the particles, whereas the spatial scale is determined by fluctuations.

Let us now compare the fluctuation and the toroidal terms for perpendicular motion. The situation here is quite different from the longitudinal one. The fluctuating particle velocity

$$
\tilde{v} \sim \frac{c \tilde{E}}{B_{0}}+v_{\|} \frac{\tilde{B}}{B_{0}} \sim \frac{\rho_{i} v_{T i}}{L_{n}}\left(1+\frac{v_{\|}}{v_{T i}} \frac{L_{n}}{q R}\right),
$$

is typically higher than the toroidal drift $u_{d}$ for both electrons and ions as

$$
\frac{\tilde{v}}{u_{d}} \sim \frac{\rho_{i} v_{T i}}{L_{n}}\left(\frac{v_{T i}^{2}}{\omega_{B i} R}\right)^{-1} \sim \frac{R}{L_{n}} \frac{v_{T}}{v_{T i}} \gg 1
$$

The amplitude of the magnetic field fluctuation, $\tilde{B}$, used in these expressions was estimated from the condition of high plasma longitudinal conductivity, $E_{\|}=k_{\|} \tilde{\phi}-\omega \tilde{A} / c \simeq 0$ :

$$
\frac{\tilde{B}}{B_{0}}=\frac{k_{\perp} \tilde{A}}{B_{0}} \sim \frac{k_{\perp}}{B_{0}} \frac{k_{\|} c \tilde{\phi}}{\omega} \sim \frac{L_{n}}{q R} \frac{\epsilon \tilde{\phi}}{T} \sim \frac{1}{k_{\perp} q R} .
$$

Estimations (5)-(11) are, of course, too rough to describe radial profiles of fluctuations, but in general they correspond relatively well to fluctuation levcls in the main region of the discharge. ${ }^{2}$

\section{Characteristics of Particles' Trajectories in Spatially Random Fields}

In this section we proceed to the analysis of particle drift trajectories of the Hamiltonian (7) which describes the system with one degree of freedom and explicit time dependence that gives rise to diffusion. ${ }^{6}$ For an understanding of the interaction mechanism between $\tilde{v}$ and $u_{d}$ in the particle motion one should analyze the equipotentials of the Hamilionian $H\left(\mathbf{r}_{\perp}\right)=$ const. that describe the integrable motion of particles in the instantaneous IIamiltonian 
$H\left(\mathbf{r}_{\perp}\right)$. The trajectories in this case approximately corresponds to the limit $\tilde{v} k_{\perp} \gg \Delta \omega, \omega$, that is close to real tokamak conditions.

Let us begin consideration in the limit $u_{d}=0$. In this case particle trajectories coincide with the equipotential lines $H\left(\mathbf{r}_{\perp}\right)=$ const. and constitute a complex structure of convective cells (Fig. 1). ${ }^{7}$ To describe the distribution of the trajectories with size percolation theory ${ }^{8}$ is used. One notes that all trajectories are closed and are generally confined to an area of two dimensional phase space of $\sim \Delta^{2}$. For small scale orbits, where $\Delta \sim \lambda \equiv k^{-1}$, the length, $L$, of the trajectory has a regular form $L \propto \Delta$. However it have been shown that large scale orbits, where $\Delta \gg \lambda$, are fractal curves that have a scaling

$$
L \sim \lambda\left(\frac{\Delta}{\lambda}\right)^{d} \gg \lambda
$$

where $d=1+1 / \nu$ is the fractal dimension of the curve and $\nu=4 / 3$ is the critical exponent derived for two dimensional case in. ${ }^{9}$

Long trajectories are most important for transport processes as was first mentioned in connection with the problem of anomalous transport in tokamaks in reference. ${ }^{10}$ An important parameter for the formation of a large convective cell is the relative equipotential altitude $h=H / H_{0}$. Large orbits appear only for $h \ll 1$. The maximal size of an $h$-level orbit is ${ }^{7}$

$$
\Delta_{h} \sim \lambda h^{-\nu} \gg \lambda
$$

and their phase space occupancy fraction. is

$$
f_{h} \sim k^{\nu} \ll 1
$$

With a small toroidal drift $\left(u_{d} \ll \tilde{v}\right)$ present trajectories change considerably. The Hamiltonian $H\left(\mathbf{r}_{\perp}\right)$ then has an average slope in the direction perpendicular to $\mathbf{u}_{d}$. Due to this slope all long trajectories with size larger than some critical value, $\Delta_{d}$, become open and head in the direction of $u_{d}$ (Fig. 2). The condition for the opening of a trajectory is determined by 
the balance of the fluctuation and drift Hamiltonian contributions ${ }^{11,12}$

$$
h_{d} H_{0} \sim u_{d} \Delta_{d}
$$

where $h_{d}$ is the relative level of an open orbit and $\Delta_{d}$ is its width. From this equation and Eq. (13) one can determine parameters for the open orbits

$$
\begin{aligned}
& h_{d} \sim\left(\frac{\tilde{v}}{u_{d}}\right)^{-1 /(\nu+1)} \ll 1, \\
& \Delta_{d} \sim \lambda\left(\frac{\tilde{v}}{u_{d}}\right)^{\nu /(\nu+1)} \gg \lambda, \\
& f_{d} \sim\left(\frac{\tilde{v}}{u_{d}}\right)^{-\nu /(\nu+1)} \ll 1,
\end{aligned}
$$

Condition (15) has a simple physical meaning. It can be rewritten as conservation of fluid flux in the direction of toroidal drift

$$
f_{d} \bar{v}_{d}=\langle\nabla H\rangle=\langle\nabla \widetilde{H}\rangle+\mathbf{u}_{d}=\mathbf{u}_{d}
$$

where $\bar{v}_{d}$ is the average directed velocity along an open trajectory

$$
\bar{v}_{d} \sim \frac{\Delta_{d}}{\tau} \sim \Delta_{d} \frac{\tilde{v}}{L} \sim \tilde{v} h_{d} \sim u_{d}\left(\frac{\tilde{v}}{u_{d}}\right)^{\frac{\nu}{1+\nu}} \gg u_{d} .
$$

One should note that whereas estimation (20) is approximate, equation (19) is precise.

The picture of trajectories described above allows for the maintenance of neoclassical effects even at high fluctuation velocities of particles $\left(\tilde{v} \gg u_{d}\right)$. Whereas the main fraction of particles circulates along closed trajectories, a small fraction $f_{d}$ moves in the direction of $u_{d}$ with the average velocity $\bar{v}_{d}\left(u_{d}<\bar{v}_{d}<\tilde{v}\right)$, so that hydrodynamic flow is the same as in the absence of fluctuations. In connection with the problem of anomalous transport in fluctuating fields such an cffect was first mentioned in. ${ }^{13}$ But the enhancement of the instantaneous drift flow $\left(\bar{v}_{d}>u_{d}\right)$ has not been previously recognized. 


\section{Neoclassical Transport Processes in Fluctuating Fields}

As have been mentioned above, diffusion emerges due to the dependence on time of the Hamiltonian (7). This time dependence is connected with the finite spectrum width of the oscillations $\left(\tau_{\omega} \sim \Delta \omega^{-1} \geq \omega_{*}^{-1}\right)$ and the variation of the $u_{d}$ direction due to collisions $\left(\eta_{1} \sim \nu_{c} / \omega_{t}^{2}\right)$ or free streaming of particles along field lines $\left(\tau_{t} \sim \omega_{t}^{-1}\right)$. The effect of the mutual influence of the toroidal drift and the fluctuations is most pronounced in the case of a narrow spectrum and weak collisions. This limit will be considered in this section, especially as in the core of the plasma the Coulomb collisions do not influence longitudinal motion and the inequality $\tau_{t} \leq \tau_{\omega}$ is typically valid. The role of collisions and broad spectrum width will be considered in the next section.

Let as consider a systems having two types of trajectories: closed, along which particles oscillate without net displacement, and open, along which particles, though oscillating, move in the direction of the toroidal drift. A new diffusion mechanism then arises that is connected with transitions between the two different types of orbits. Due to the time dependence of the Hamiltonian, orbits in a given topology have finite lifetime. During the lifetime in an open trajectory, $\tau_{d}$, particles move in the direction of toroidal drift for a distance $\Delta r \sim \bar{v}_{d} \tau_{d}$. Afterwards, the topology of the trajectory alters and the particles become circulating in a quasi-closed orbit. Eventually an inverse process occurs, and a closed trajectory converts to an open one and the particles again begin moving along the toroidal drift, but in a random direction with respect to their initial displacement (Fig. 3). Such a random walk leads to diffusion with a coefficient

$$
D \sim f_{d} \frac{\Delta r^{2}}{\tau_{d}} \sim f_{d} \bar{v}_{d}^{2} \tau_{d} \sim u_{d}^{2} \tau_{d} \frac{\bar{v}}{u_{d}}
$$


The trajectory life time can be estimated from the relation ${ }^{14}$

$$
\tau_{d} \sim \frac{H}{d H / d t} \sim \frac{h_{d} \tilde{v} \lambda}{u_{d} \Delta_{d} \omega_{t}} \sim \omega_{t}^{-1} .
$$

Substituting $\tau_{d}$ into the formula (21) yields the ion diffusion coefficient in tokamak3 with account of both fluctuation fields and toroidal drifts,

$$
D=C_{0} \frac{u_{d}^{2}}{\omega_{t i}}\left(\frac{\tilde{v}}{u_{d}}\right)^{4 / 7} \sim \frac{u_{D}^{2}}{\omega_{t i}}\left(\frac{R}{L_{n}}\right)^{4 / 7} \geq D^{p \ell} \sim \frac{u_{d}^{2}}{\omega_{t i}}
$$

Here only ion parameters are used as we expect neoclassical effects to be more important for the ion plasma component. It can be seen from this expression that the diffusion coefficient, in the presence of oscillations with sufficiently high amplitude $\left(\tilde{v}>u_{d}\right)$, is larger than the neoclassical plateau value by about a factor of $\left(\tilde{v} / u_{d}\right)^{4 / 7} \sim\left(R / L_{n}\right)^{4 / 7}$. Moreover this coefficient does not depend on the collisional frequency and therefore applies even in the banana regime, whereas the neoclassical diffusion decreases proportionally to the collision frequency.

The theoretically predicted diffusion coefficient given in $\mathrm{Eq}$. (23) was tested by numerical simulations. Results of a numerical experiment ${ }^{15}$ are presented in Fig. 4 and confirm the dependence of the diffusion coefficient on the fluctuation amplitude $D \sim\left(\tilde{v} / u_{d}\right)^{4 / 7}$ at $\tilde{v}>u_{d}$ within the exponent error bars \pm 0.1 . The sharp decrease of the diffusion coefficient at $\tilde{v}<u_{d}$ correspon ls to the vanishing of closed orbits. The simulation also allows the determination of the numerical constant in Eq. (23) $C_{0}=0.13$.

It is necessary to note that the estimate of the trajectory life time (22) implies a randomness in the time dependence of the Ilamiltonian. In the problem under consideration the type of randomness differs from that used in Ref. 14. As the explicit time depencence of Hamiltonian is regular $\left(\theta(t)=\omega_{t} t\right)$, for the decorrelation mechanism one uses that the particles moves in a spatially random field of oscillations. Ilence the displacement term, 
$\mathbf{r}_{\perp}(t)$, is random in the Hamiltonian time derivative,

$$
\frac{d H}{d t}=-\omega_{t} u_{d}(x \sin \theta(t)-y \cos O(t))
$$

One should also note that the estimation $d H / d t=\Delta_{d} u_{d} \omega_{t}$ used in Eq. (22) is valid only when the characteristic decorrelation time, $\tau_{d}$, is approximately the time for particles to go around a characteristic orbit width, $\Delta_{d} / \bar{v}_{d}$. Using Eqs. (17), (20) and (22) this condition can be rewritten as an equality of neoclassical trajectory size $\Delta r=u_{d} / \omega_{t} \sim q \rho_{i}$ and the characteristic spatial scale of fluctuations is $\lambda \sim k_{\perp}^{-1}$. Taking into account that for typical drift turbulence the wavelength scale $\lambda \sim \rho_{i}$, one can see that condition $\lambda \sim \Delta r$ is indeed consistent for ions $\left(\lambda \sim \rho_{i} \sim \Delta r \sim q \rho_{i}\right)$ in the plasma column core $(q \sim 1)$.

Consideration of the general case is a considerably more difficult task. ${ }^{15}$ In this short article it is only possible to note that for $\tilde{v} / \lambda>\omega_{t}$ diffusion mechanism is weakly influenced by the parameter $\lambda \omega_{t} / u_{d}$. The transport coefficient valid in this parameter interval for both ions and electrons can be written as

$$
D_{0}=C_{0} \lambda u_{d}\left(\frac{\tilde{v}}{u_{d}}\right)^{4 / 7}
$$

At higher frequencies $\omega_{t} \lambda>\tilde{v}$ the toroidal drift becomes too fast for a spatially random IIamiltonian, $\widetilde{I}\left(\mathbf{r}_{\perp}\right)$, to be the reason for trajectory decorrelation $\left(\tilde{v} / \omega_{t}<\lambda\right)$. Therefore if the finite spectrum width of fluctuations is not taken into account, the motion of the majority of particles at $\lambda>\tilde{v} / \omega_{t}$ is adiabatic,

$$
H_{\mathrm{eft}}=\frac{\omega_{t}}{2 \pi} \oint \widetilde{H} d t
$$

and the diffusion coefficient drops considerably with respect to Eq. (25). Such a dependence of diffusion cuefficient on $\lambda \omega_{t} / u_{d}$ is illustrated by the fall-off of the high frequency response in the numerical results shown in Fig. 5. 


\section{Discussion}

In this section we discuss the influence of other processes on the modification of neoclassical effects in tokamaks.

Collisions influence longitudinal motion when $\eta_{\|} \equiv \nu_{c} / \omega_{t}^{2}>\omega_{t}$ (neoclassical PfirschSchlüter regime). In this regime the direction of toroidal drift $u_{d}$ alters in a time scale $\eta_{1}$. Moreover, due to the nature of the collision process, the toroidal drift direction changes randomly. Therefore the decorrelation time $\eta_{\mid}$should be substituted in Eq. (21) instead of trajectory life time $\eta_{\mid}$to estimate the diffusion coefficient in the Pfirsch-Schlüter regime

$$
D \sim f_{d} \bar{v}_{d}^{2} \eta_{\mid} \sim q^{2} \rho^{2} \nu_{c}\left(\frac{\tilde{v}}{u_{d}}\right)^{4 / 7} \sim D^{\mathrm{PS}}\left(\frac{R}{L_{n}}\right)^{4 / 7} .
$$

From this expression one can see that in the Pfirsch-Schlüter regime, as well as in low collisionality cases, high amplitude fluctuations considerably increase the transport coefficient with respect to the neoclassical value.

Transport processes, due to the broad spectral width in a two-dimensional system, have been considered $i^{14}$ In this case $\widetilde{H}$ is randomly dependent on time and one should use in Eq. (22), $d H / d t \sim H_{0} / \Delta \omega$, to estimate the additional trajectory lifetime limitation. Under the conditions all long trajectories are destroyed no matter if they are open or closed and then diffusion is independent of $u_{d}$. For convenience of comparison with Eq. (25) the transport coefficient obtained $\mathrm{in}^{14}$ can be represented in the following form

$$
D_{\omega} \sim \Delta \omega \lambda^{2}\left(\frac{\tilde{v}}{\Delta \omega \lambda}\right)^{7 / 10}=\lambda u_{d}\left(\frac{\tilde{v}}{u_{d}}\right)^{7 / 10}\left(\frac{\lambda \Delta \omega}{u_{d}}\right)^{3 / 10} .
$$

Formally this coefficient exceeds $D_{0}(25)$ when

$$
\Delta \omega \geq u_{d} / \lambda\left(\frac{\tilde{v}}{u_{d}}\right)^{-3 / 7} .
$$

However the dependence of $D_{\omega}$ on $\Delta \omega$ is weak and the difference of the $\tilde{v} / u_{d}$ dependence compared with Eq. (25) is extremely small. Hence even for the maximum spectrum width 
for drift fluctuations in a tokamak $\Delta \omega \sim \omega_{t}$, there is almost no change in diffusion coefficient with respect to $D_{0}$ (Fig. 4).

Both effects discussed above only increase diffusion in comparison to Eq. (25). The question also arises whether there are other mechanisms to inhibit the diffusion caused by the toroidal drift in the presence of fluctuations. Such processes are needed to describe $H$-mode regimes $^{16}$ as the diffusion estimate in Eq. (25) seems too high to agree with experiment. One of the candidates for such a process is plasma rotation or, to be more precise, the difference in poloidal rotation of the plasma and the oscillations. To see this let us transform Hamiltonian (7) to the frame rotating with the fluctuating fields. As a result an additional term $u_{p} x\left(u_{p}\right.$ being the difference of poloidal rotation speeds of the plasma and oscillations) will appear in Hamiltonian

$$
H\left(\mathbf{r}_{\perp}, t\right)=\widetilde{H}\left(\mathbf{r}_{\perp}, t\right)+u_{d}(x \cos \theta(t)-y \sin \theta(t))+u_{p} x
$$

This additional term will lead to the shrinkage of all trajectories in the radial direction. It is evident that at $u_{p} \geq \tilde{v}$ long trajectories will disappear together with the diffusion that could be induced. The disappearance of diffusion at $u_{p} / \tilde{v} \geq 1$ is illustrated by the numerical result in Fig. 6. One should note that velocity difference $u_{p}$ can not be associated with a constant radial electric field but it may take place due to pressure gradients or high poloidal rotational shear.

In addition to the mutual influence of $u_{d}$ and $\tilde{v}$ in perpendicular motion there is the effect of the fluctuations on the longitudinal motion. Although for the majority of particles turbulent acceleration is not important, there is a group of resonant particles with $v_{\|}=v_{i} \equiv$ $\omega / k_{\|}$that interact with the fuctuations exchanging toroidal momentum with the resonant electromagnetic waves. Such an exchange can lead to the additional longitudinal viscosity and typically increases neoclassical effects. ${ }^{17,18}$

We now summarize how ncoclassical effects manifest themselves under typical tokamak 
conditions where the fuctuation velocities $\tilde{v}$ are higher than the toroidal drift velocity $u_{p}$. Two features should be emphasized: (i) as the longitudinal particle motion is weakly influenced by drift turbulence where $k_{\|} \sim / q R \ll k_{\perp}$, the toroidally trapped particle number does not change; (ii) the perpendicular trajectories of the majority of particles is primarily determined by fluctuations, but the overall diffusion is determined by a small particle fraction that move in the direction of the toroidal drift with sufficiently high velocities to produce the same hydrodynamical flow as in the absence of fluctuations. The diffusion coefficient $D_{0}$ given in Eq. (25) typically considerably exceeds the neoclassical value in all collisionality regimes. The coefficient $D_{0}$ is weakly dependent on amplitude and spectrum of oscillations for typical tokamak conditions $u_{d} \leq \tilde{v} \leq 10 u_{d}$ and $\Delta \omega \leq \omega_{t}$. At the same time some other neoclassical effects are weakly dependent on this process and can become even more pronounced if the resonant longitudinal interaction is taken into account. 


\section{References}

1. Groebner, R.J., Pfeiffer, W., Biau, F.P. et al., Nucl. Fusion 26, 543 (1986).

2. Liewer, P.C., Nucl. Fusion 25, 543 (1985).

3. Mescrvay, E., Bitter, M., and Daudhney, C., Nucl. Fusion 24, 3 (1984).

4. Adarns, J.M., Altmann, H, Appruzzese, G. et al., Plasma Physics and Controlled Nuclear Fusion Research (Proc. 12th Int. Conf., Nice, 1988), Vol. 1, IAEA, Vienna, 1989 , p. 41 .

5. Kadomtsev, B.B., Plasma Turbulence, (Academic Press, New York, 1965).

6. Lichtenberg, A.J., and Lieberman, M.A., Regular and Stochastic Motion (SpringerVerlag, New York, 1983).

7. Isichenko, M.B., Kalda, J., Tatarinova, E.B., Telkovskaya, and O.V., Yankov, V.V., Zh. Eksp. Teor. Fiz. 96, 913 (1989) [Sov. Phys. JETP 69, 517 (1989)].

8. Feder, J., Fractals (Plenum Press, New York, 1988).

9. Saleur, H., and Duplantier, B., Phys. Rev. Lett. 58, 2325 (1987).

10. Kadomtsev, B.B., and Pogutse, O.P., in Plusma Physics and Controlled Nuclear Fusion Research (Proc. 7th Int. Conf., Innsbruck, 1978), Vol. 1, IAEA, Vienna, 1979, p. 649.

11. Trugman, S.A., Phys. Rev. B 27, 7539 (1983).

12. Isichenko, M.B., and Kalda, J., Proc. 17th EPS Conf. on Contr. Fusion and Plasma Heating (Amsterdam, 1990), Part II 14B, 667. 
13. Zel'dovich Ya.B. Pis'ma v Zh. Eksp. Teor. Fiz. 38, 51 (1983) [Sov. Phys. JETP Lett. 38, 57 (1983)].

14. Gruzinov, A.V., Isichenko, M.B., and Kalda, J., Zh. Eksp. Teor. Fiz. 97, 476 (1990) [Sov. Phys. JETP 70, 263 (1990)].

15. Smolyakov, A.I., and Yushmanov, P.N., submitted to Nuclear Fusion.

16. Wagner, F. et al., Phys. Rev. Lett. 28, 1408 (1982).

17. Yushmanov, P.N., Pis'ma Zh. Eksp. Teor. Fiz. 31, 88 (1980) [Sov. Phys. JETP Lett. 31, 80 (1980).

18. Connor, J.W., and Taylor, J.B., Comm. Plasma and Contr. Fus. 21, 37 (1987). 


\section{Figure Captions}

1. Particle trajectories in the spatially random potential with scale $\lambda$.

2. Particle trajectories in the random potential with the addition of the toroidal drift $u_{d}=\tilde{v} / 30$. Spatial potential distribution and initial conditions of trajectories are the same as in Fig. 1.

3. An example of the trajectory for the frequency of the toroidal velocity direction variation $\omega_{t}=u_{d} / \lambda$.

4. The dependence of the diffusion coefficient on fluctuation amplitude for $\omega_{t} \lambda / u_{d}=$ 1: closed circles $-\Delta \omega=0$, open circles $-\Delta \omega=\omega_{t}$, solid line represents percolation estimate given by Eq. (25).

5. The dependence of the diffusion coefficient on particles rotation frequency along magnetic field lines.

6. The dependence of diffusion coefficient on the difference of poloidal rotation velocity of particles and fluctuations. 


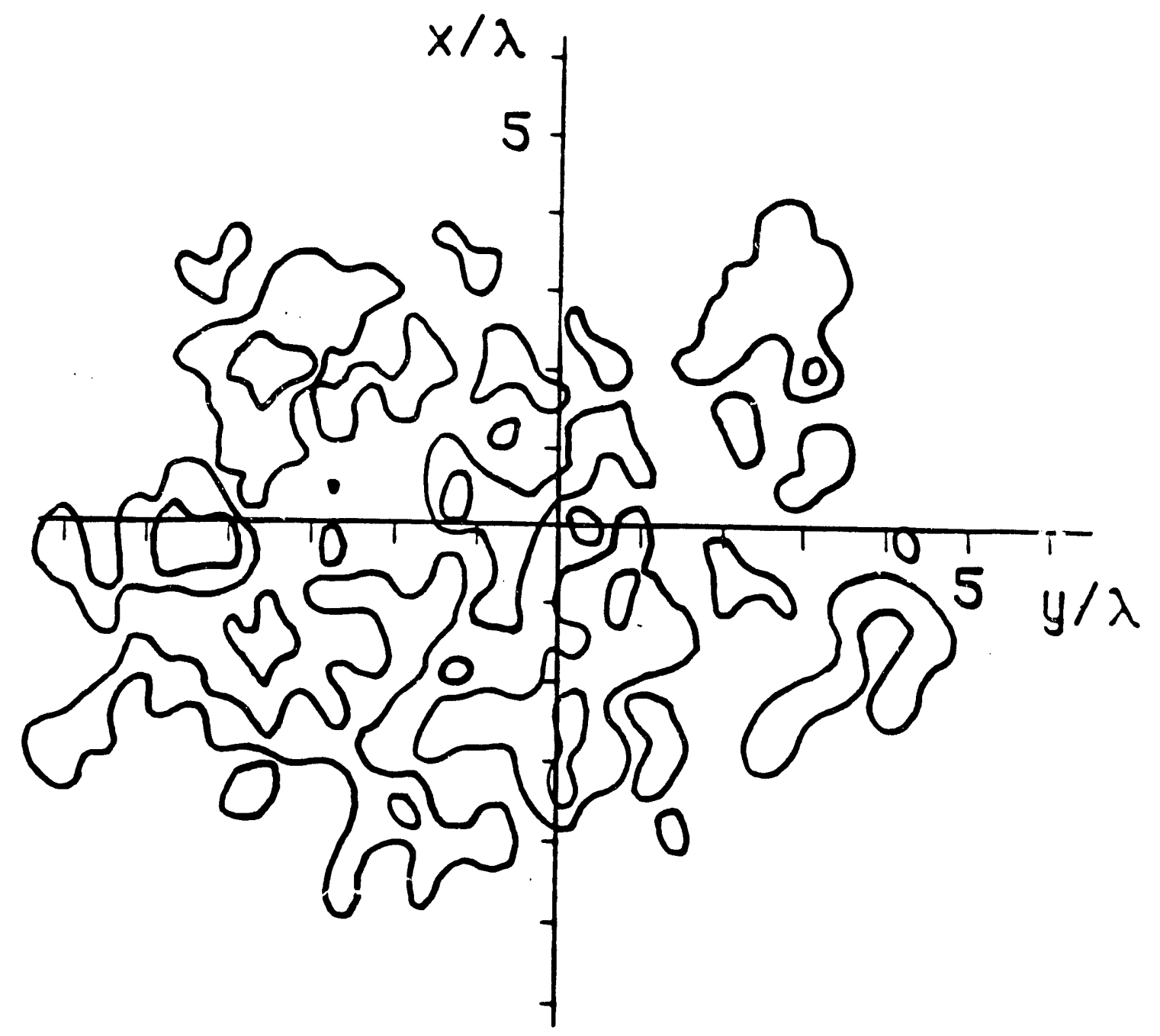

Fig. 1 


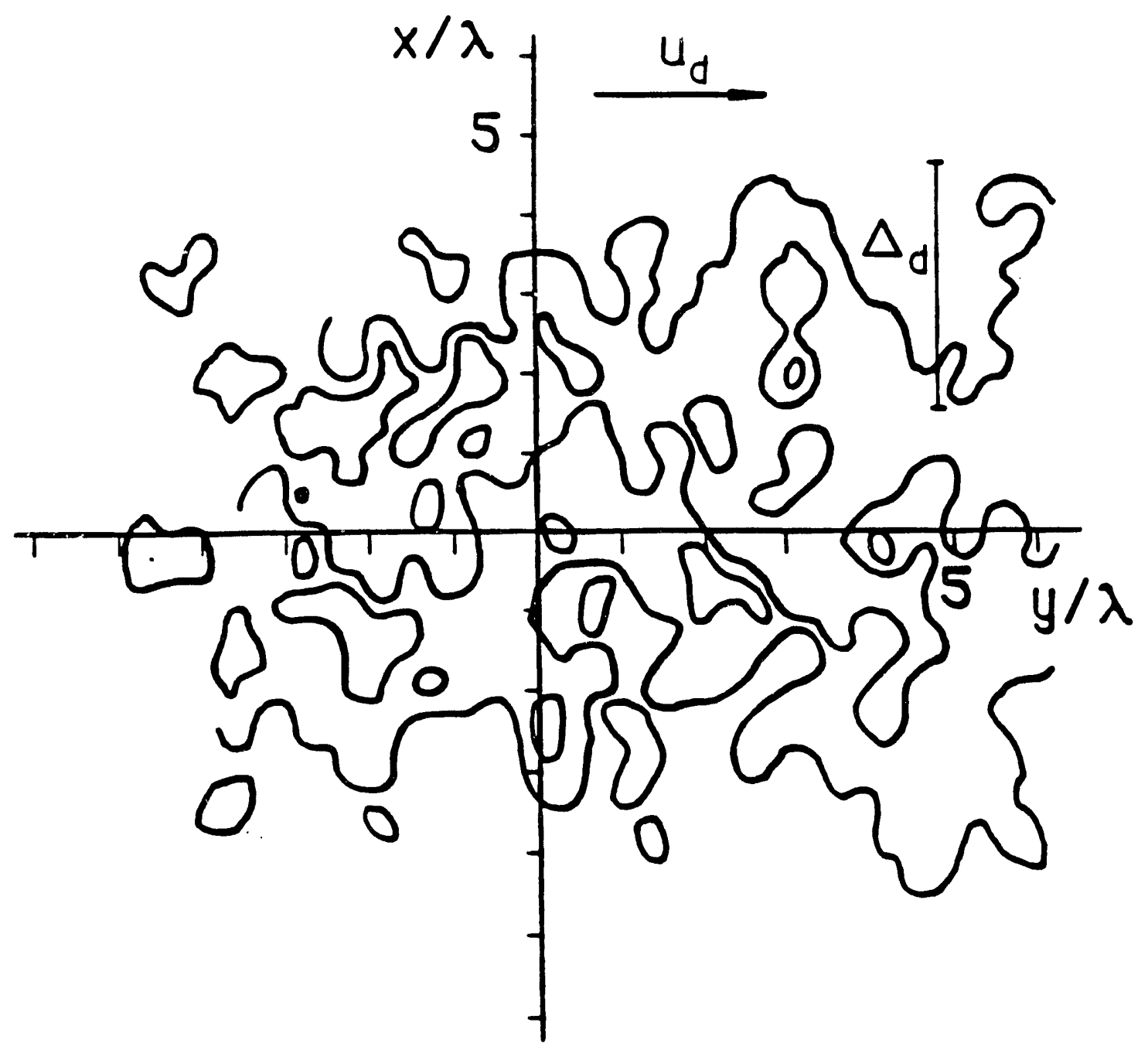

Fig. 2 


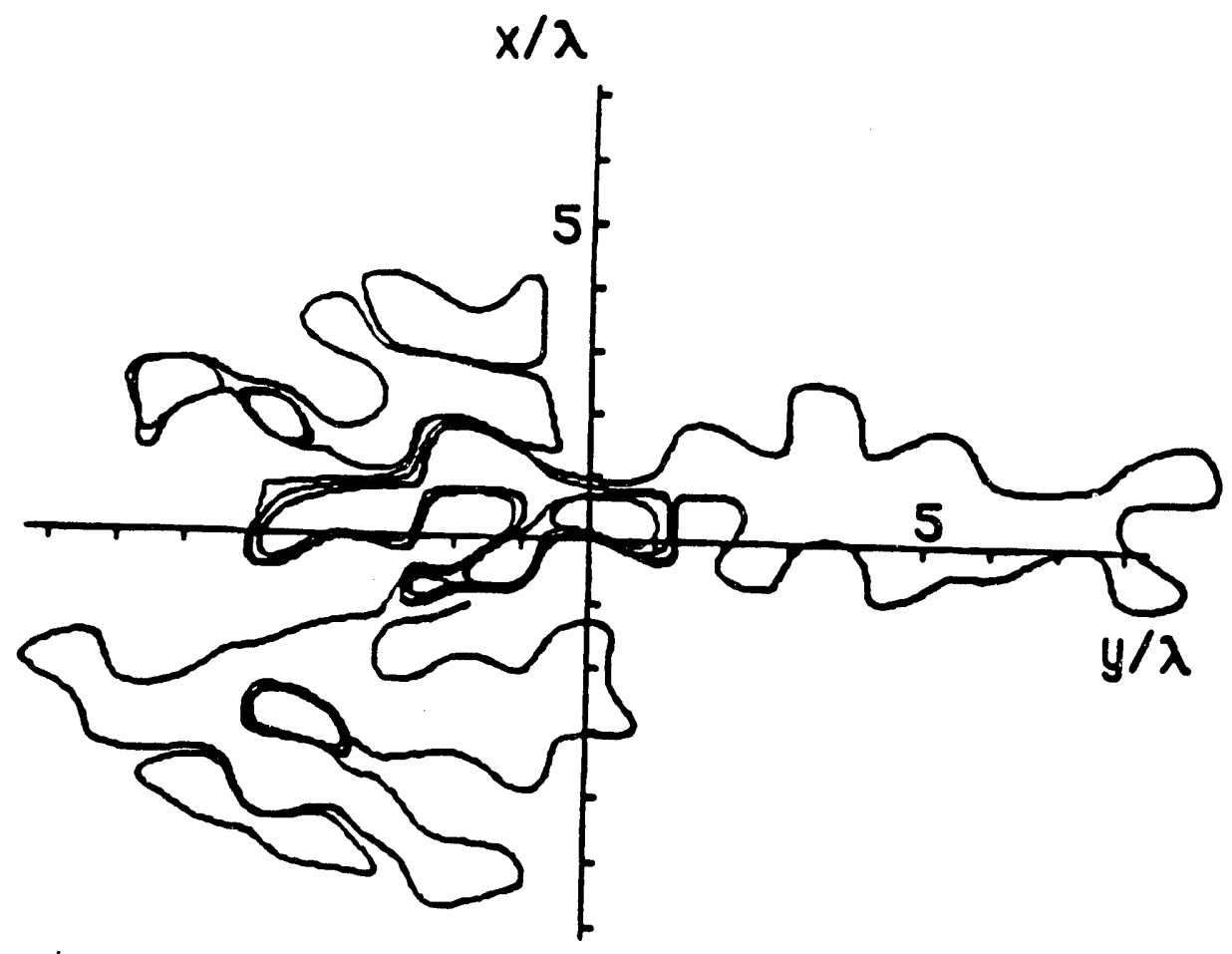

Fig. 3 


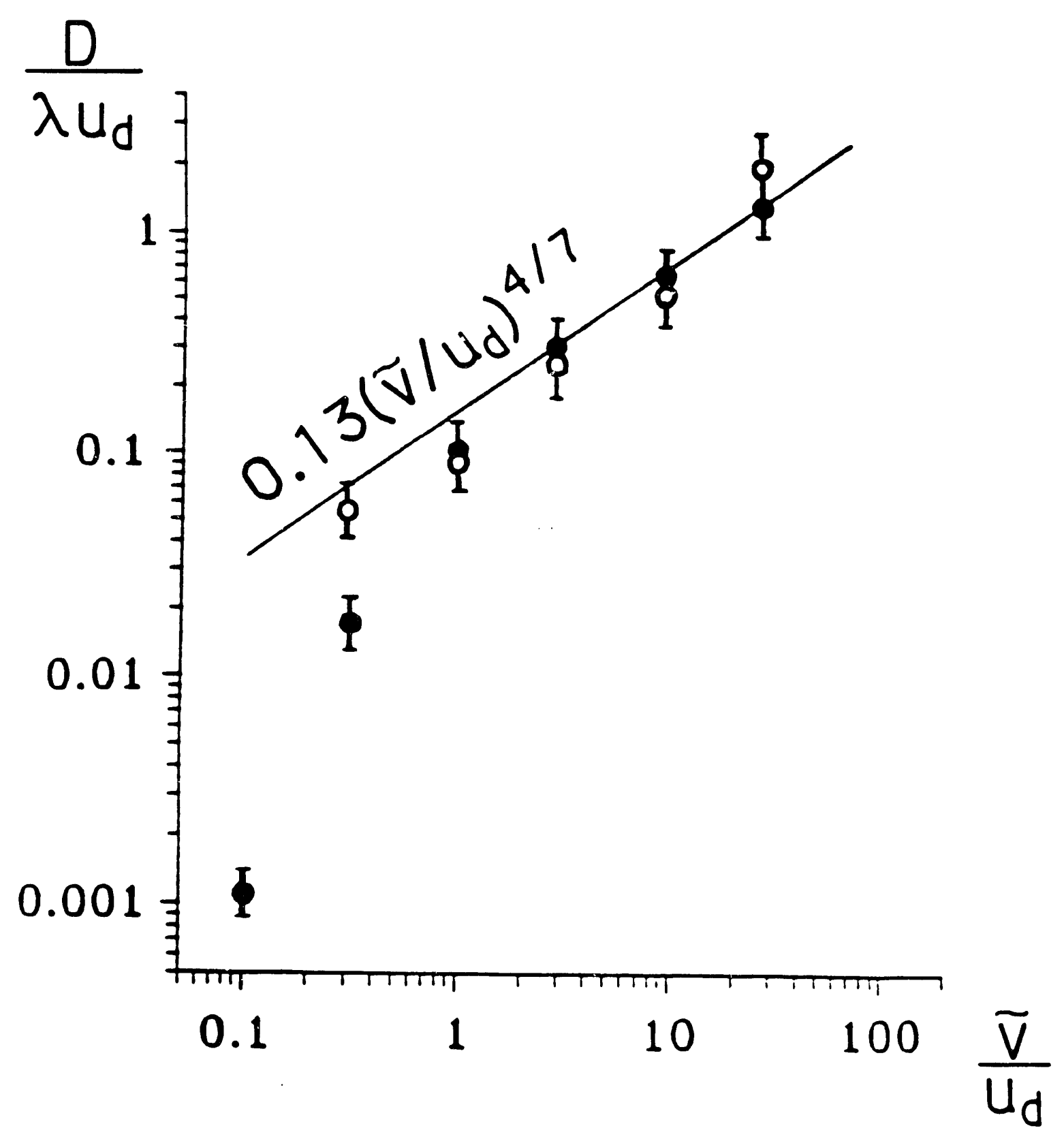

Fig. 4 


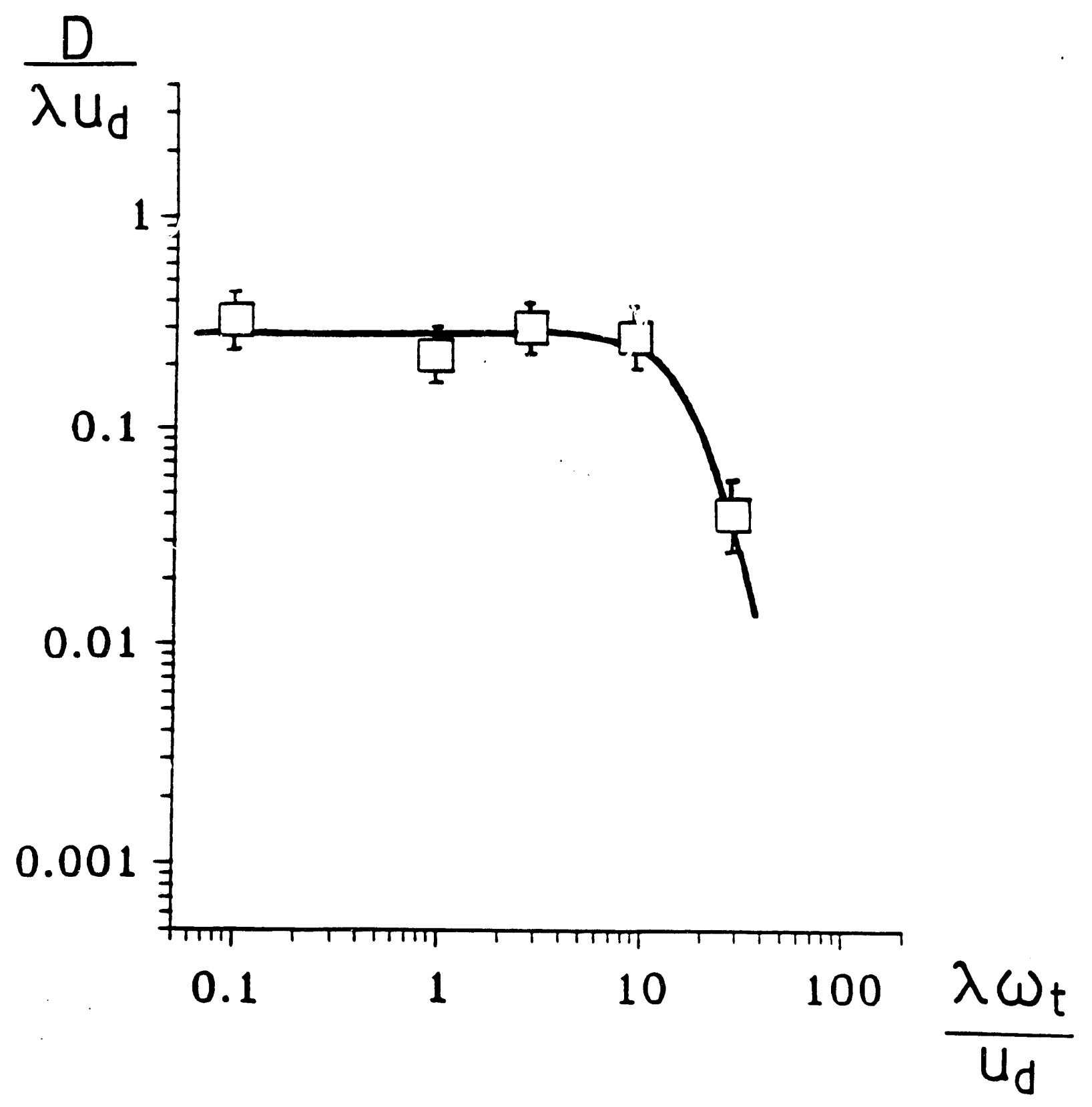

Eig. 5 


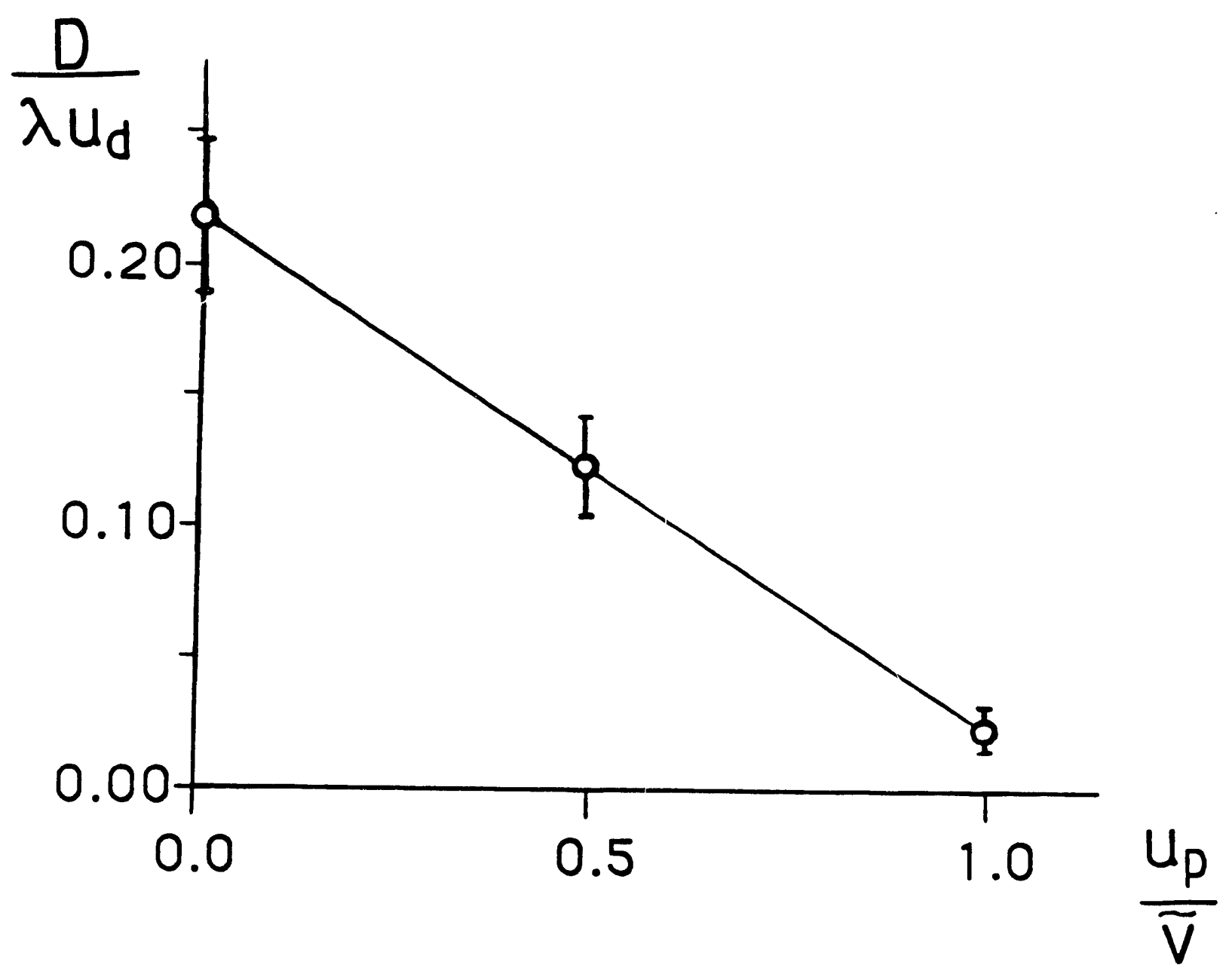

Fig. 6 

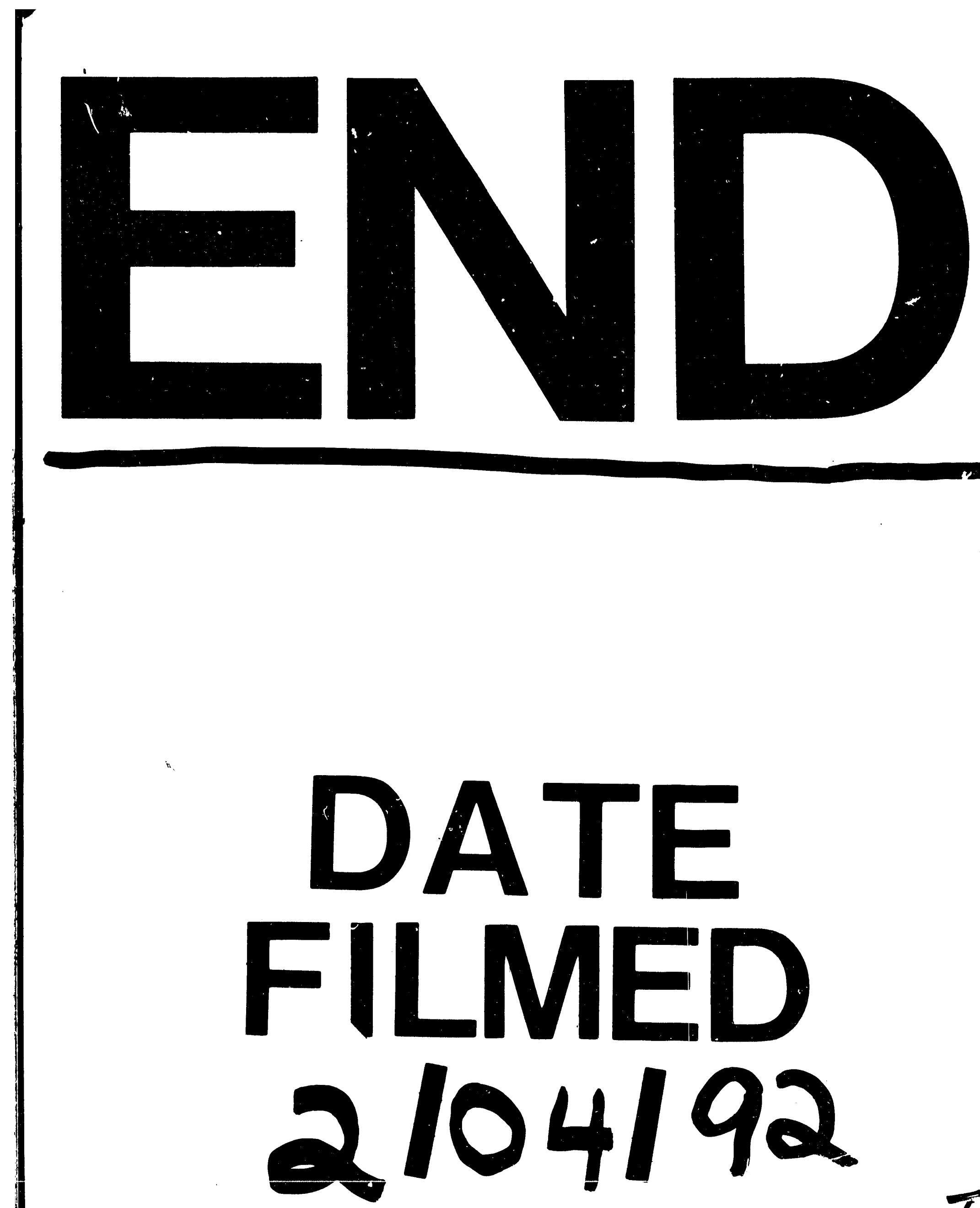

$I$ 
